\title{
Quality of Service Oriented Access Point Selection Framework for Large Wi-Fi Networks
}

\author{
Alessandro Raschellà, Faycal Bouhafs, Mirghiasaldin Seyedebrahimi, Michael Mackay, Qi Shi
}

\begin{abstract}
This paper addresses the problem of Access Point (AP) selection in large Wi-Fi networks. Unlike current solutions that rely on Received Signal Strength (RSS) to determine the best AP that could serve a wireless user's request, we propose a novel framework that considers the Quality of Service (QoS) requirements of the user's data flow. The proposed framework relies on a function reflecting the suitability of a Wi-Fi AP to satisfy the QoS requirements of the data flow. The framework takes advantage of the flexibility and centralised nature of Software Defined Networking (SDN). A performance comparison of this algorithm developed through an SDN-based simulator shows significant achievements against other state of the art solutions in terms of provided QoS and improved wireless network capacity.
\end{abstract}

Index Terms-Wi-Fi networks, Access point selection, Software Defined Network.

\section{INTRODUCTION}

$\mathrm{T}$ HE last few years have witnessed a significant increase in the use of portable computing devices such as smartphones, tablets and laptops. The popularity of these devices and the emergence of a range of innovative mobile applications and online services are driving the demand for more reliable wireless communication connectivity. Despite the maturity of mobile radio technologies such as $3 \mathrm{G}$ and $4 \mathrm{G}$, Wi-Fi still represents a cheaper, faster, and more reliable communication alternative for many wireless users. In addition to the wireless users, operators are now looking to offload data from their cellular networks to Wi-Fi networks. Moreover, the use of Wi$\mathrm{Fi}$ will also play a key role in future $5 \mathrm{G}$ systems where the integration of different Radio Access Technologies (RATs) will be used to maximize users' Quality of Experience (QoE). In fact, $4 \mathrm{G}$ networks are already evolving towards $5 \mathrm{G}$ to include various small nodes, such as pico and femto cells with a similar coverage range to $\mathrm{Wi}-\mathrm{Fi}$, as well as $\mathrm{Wi}-\mathrm{Fi}$ networks [1]. This integration introduced in the context of the $5 \mathrm{G}$ is also known as network densification.

The popularity of Wi-Fi technology has also made its way into the work place and public spaces such as airports, train stations, and university campuses. Large scale Wi-Fi networks are built by deploying Radio Frequency (RF) overlapping WiFi Access Points (APs), in order to guarantee good signal

Manuscript submitted on August $9^{\text {th }}, 2016$.

This work has received funding from the European Union's Horizon 2020 Research and Innovation Programme under Grant Agreement no. 644262 as part of the Wi-5 project. coverage and redundant connectivity to the user. These modern enterprise Wi-Fi networks are managed by IT officers who are constantly facing the challenge of satisfying the increasing demand of their network users for more capacity and better connectivity. Unlike wired networks, where the size of the network is fixed and the amount of traffic can be predicted, enterprise Wi-Fi networks are very dynamic as wireless users can join and leave at any moment. More importantly, the traffic within these networks is characterised by heterogeneous Quality of Service (QoS) demands and different transmission rates, as each wireless user might be running a different application. Moreover, these demands are increasing over time as more bandwidth-hungry services are introduced. However, since the Wi-Fi spectrum is a finite resource, a significant increase in the wireless traffic will ultimately result in congestion within the network, affecting the overall quality of coverage, and reducing the overall performance. We are already seeing the impacts of this today.

Upon joining a Wi-Fi network, wireless users are usually associated to the AP that provides the best signal coverage within the enterprise Wi-Fi network. However, such an approach does not consider QoS requirements as a factor, which could affect the overall network performance. A study published in [2] shows that the AP association approach based on Received Signal Strength Indicator (RSSI) and recommended by the IEEE 802.11 standard might negatively affect the overall network spectrum efficiency and capacity.

Although there are a number of Wi-Fi network management solutions available in the market, most of these solutions are generally proprietary, which makes it difficult to extend their functionality and improve their flexibility such that spectrum efficiency and QoS requirements can be considered. More recently, Software-Defined Networking (SDN) [3] has emerged as an open, efficient and flexible network management concept for large networks. By decoupling the control plane from the data plane, SDN can centralise network management operations in a single entity, often referred to as a controller. Due to its flexibility, the SDN concept is also currently being adopted for wireless network management, including Wi-Fi networks.

Building on this latest development, in this paper we propose a dynamic AP selection approach implemented in a centralised framework based on the SDN concept, in which the controller

A. Raschellà, F. Bouhafs, M. Seyedebrahimi, M. Mackay, and Q. Shi are with the Department of Computer Science, Liverpool John Moores University (LJMU), Liverpool, UK (e-mail: \{A.Raschella, F.Bouhafs, M.Seyedebrahimi, M.I.Mackay, Q.Shi\}@ljmu.ac.uk). 
managing the Wi-Fi network selects the most suitable AP for a specific application. The proposed approach is based on a novel AP selection algorithm that calculates and assigns a performance metric to each AP, called Fittingness Factor (FF), which is a function addressing the suitability of the available spectrum resources to the application requirements. Before assigning an AP, the algorithm calculates another parameter, called Network Fittingness Factor that takes into account the QoS requirements of a wireless user joining the network, the current network capacity, and the quality of the connectivity provided to the remaining wireless users. Based on this information, the proposed strategy determines the most suitable AP for the required application in terms of the Network Fittingness Factor.

The AP selection algorithm presented in this paper extends our previous work proposed in [4] in several facets. Specifically, with respect to previous work in this area found in the literature and our previous work published in [4], the AP selection algorithm relies on an extended version of the socalled Network Fittingness Factor metric. In this paper, such a metric jointly addresses: (i) the QoS requirements of a flow joining the network; (ii) the bandwidth efficiency; and (iii) the QoS requirements of the other flows active in the network. Compared to [4], we provide the following new contributions:

- A knowledge database is introduced in the SDN-based controller in order to keep track of all the flows that are connected to the network. As we will explain, the information stored in the knowledge database will be crucial for the AP selection algorithm to safeguard the QoS requirements of all the active flows in the network each time a new flow needs an AP association.

- The SDN-based framework is enhanced with the inclusion of an innovative network configuration mechanism to address the optimal RF channel assignment configuration in terms of interference management and provided spectrum efficiency across all the Wi-Fi network [5].

- In terms of assessment, we have widely strengthened the performance analysis campaign including flows requiring different bit rates, new performance metrics and experiments to estimate the effectiveness of our algorithm. Moreover, we have included a further reference algorithm recently developed and based on the same centralised approach, which relies on SDN [6], to demonstrate how our proposal improves the performance of previous works on the same topic.

The rest of the paper is organized as follows: in Section II we provide a comprehensive analysis of the state of the art in the context of AP selection and our main purposes. In Section III we present our SDN-based Wi-Fi management framework, and the assumptions made while designing our proposed AP selection approach. In Section IV, we formulate the AP selection problem, and describe our AP selection algorithm based on the FF concept. In Section V we present the simulation model we used to evaluate our algorithm. The evaluation results are presented in Section VI. Finally, Section VII provides our concluding remarks and future works.

\section{STATE OF THE ART AND PAPER CONTRIBUTIONS}

The problem of AP selection has been addressed extensively in the literature, with many contributions focusing on wireless user devices to initiate the selection process. There are different ways to classify the existing works in this area. For instance, AP selection can be classified as distributed [7]-[17] or centralised [6], [18]-[21] approaches. With distributed solutions, such as game theory strategies [9]-[11], neural network [12], or cross-layer approaches [15]-[17], a wireless device usually gathers performance related measurements from the network before selecting the most suitable AP according to a specific metric. Other more centralised approaches rely on the global view obtained from the network controller to decide the most suitable AP. Although incurring more overhead, these centralised approaches tend to be more efficient, especially in large Wi-Fi networks, since the central controller is able to not only obtain a more accurate view of the state of the whole network, but also apply load balancing to avoid congesting certain APs. These approaches may also be classified based on their purposes. In the next subsections we will provide an exhaustive analysis of the main works on AP selection found in the literature, which are classified based on their goals, and then state our motivations and new contributions.

\section{A. AP selection based on performance metric maximization}

The majority of the AP selection techniques have the only objective to maximize a certain metric such as the throughput [7], [8], [10], [12], and [20], the bandwidth efficiency [9], or the minimum attenuation due to path-loss [11].

Specifically, in [7], the authors propose an AP association metric called Estimated aVailable bAnd-width (EVA). This metric associates a wireless user with the AP that provides the maximum achievable throughput. In [8], the authors propose an approach to select the AP to maximise the achievable throughput for a Wi-Fi user based on the number of users already associated with it and the data rates these users achieve.

In [9], the authors investigate the AP selection problem with variable channel-width Wireless Local Area Networks (WLANs) using an evolutionary game theoretical approach. In this work, the authors assume that all the stations or devices adopt the most efficient Modulation and Coding Schemes (MCSs) to achieve their highest bandwidth.

In [10], the author formulates the AP selection problem as a non-cooperative game where each user tries to maximize its utility function, defined as the throughput reward minus the fee charged by the AP. In [11], the authors also formulate the AP selection problem as a game where players are mobile wireless users who choose radio APs to connect to the network based on the Signal to Interference plus Noise Ratio (SINR). In [12], the authors propose a decentralised scheme that considers a neural network where the mobile devices are able to select the AP that is expected to yield the best throughput.

In [20], the authors present a centralised AP selection algorithm based on a local search method. This approach aims to achieve optimal average and minimum throughputs used as base measures for decentralised algorithms. 


\section{B. AP selection based on multiple metrics}

Other contributions take into account multiple metrics like in [13]-[17], and [19]. For instance, a utility-based AP selection approach that takes into account a wide range of interests and goals is introduced in [13]. In detail, the authors first discuss how the majority of the AP selection strategies analysed in the literature aim at maximizing the throughput, and how only a few solutions consider different objectives such as delay and other QoE metrics. Then, they propose a utility-based technique, which takes into consideration a set of metrics for QoE, and compare it against the standard RSSI-based AP selection algorithm. The work presented in [14] studies the dynamics among end-users and network operators in the processes of network selection and resource allocation based on non-cooperative game theory.

The work presented in [15] considers the benefits of adopting a cross-layer approach in AP association. In this work, the authors propose an AP selection process based on a metric that indicates the expected throughput when associated with an AP, using combined information obtained from the physical and MAC layers. PHMIPv6, a fast hand-off mechanism presented in [16], is another cross-layer approach that uses information obtained from the MAC and network layers in order to predict which AP minimises the handoff delay time and the packet loss rate. In [17], the authors describe a set of hand-off processes that use different AP selection mechanisms based on either a single metric such as the Signal to Noise Ratio (SNR) or the traffic load, or on a cross-layer design by combining the information from different layers. In [19], the authors present Wifi-Reports, a collaborative service that provides clients with historical information to improve AP selection.

\section{AP selection based on Load Balancing}

Finally other papers focus on a load balance among the APs composing a Wi-Fi network [6], [18], and [21]. In detail, in [6] the authors present an AP selection algorithm, which associates each new flow with the least loaded AP, which provides a sufficient RSSI based on the QoS requirements. In [18] the authors demonstrate that in small-scale networks, the performance of centralised approaches can often outperform RSSI-based and decentralised solutions. Several AP association algorithms have also been proposed to avoid load imbalanced situations among APs, which might degrade the network performance. For instance, in [21] the authors firstly review existing solutions on the load balancing problem in IEEE 802.11 networks, and then conduct experiments which demonstrate the benefits of load balancing solutions by comparing network performance with and without the load distribution schemes.

\section{Our motivations and novel contributions}

A significant shortcoming in all the metrics formulated in the above contributions is that they consider that all users are the same where, in reality, each user connected to the $\mathrm{Wi}-\mathrm{Fi}$ network is running an online application or accessing a service with specific QoS requirements that may differ from one user from another. Since the capacity of the Wi-Fi network is limited, it is necessary to devise an association strategy that takes into account the suitability of each traffic with a specific $\mathrm{AP}$ and introduces prioritisation according to the class of the traffic. A centralised approach will be an ideal candidate as it can obtain an accurate view of the entire network status. However, unlike the current centralised solutions, its AP selection strategy needs to provide fine-grained control of the network such that it can implement per-flow associations.

The introduction of SDN allows us to rethink flow and QoS managements in an efficient and flexible way. In SDN, the network control is decoupled from the forwarding plane and centralised in a controller. This centralised management approach allows operators to program large networks through the OpenFlow protocol [22]. From its first specifications, OpenFlow considers QoS as a part of its operations. Several contributions have shown the benefits of dynamic resource allocation and queue assignments using SDN and OpenFlow [22]. For instance, the work presented in [3] shows that changing the queue assignment of video flows based on the currently buffered playtime avoids stalling.

In a similar way, SDN could help to implement QoS management and efficient resource allocation in large $\mathrm{Wi}-\mathrm{Fi}$ networks with dense and heterogeneous users' demands. There have been a number of contributions that tried to extend SDN to wireless networks, including Wi-Fi [23]-[26]. Contributions such as OpenRoads [23], OpenSDWN [24], EmPOWER [25] and Odin [26] build new mechanisms on top of OpenFlow in order to support mobility, virtualization, and Service Set IDentifier (SSID) management.

The use of SDN to address AP selection in Wi-Fi networks has been recently proposed in [4] and [6]. In [4] we present the first version of our AP selection approach based on SDN. Specifically, we have introduced the FF concept to allow the controller to associate the most suitable AP to a device. In [6] the authors propose the use of a dynamic AP selection algorithm implemented in a SDN-based framework. In this work, the devices receive network resource-related statistics from the SDN controller, which guide the client device to associate itself with the best available AP. This association is based on the received statistics that jointly consider the network load in terms of the AP bandwidth and RSSI value.

The AP selection algorithm proposed in this paper takes advantage of these recent developments to address the aforementioned problems with QoS and spectrum efficiency in dense Wi-Fi networks. Therefore, this solution provides an approach that is also in line with the network densification problem introduced in the context of the $5 \mathrm{G}$. The aims of this paper can be summarized as follows:

- To provide an innovative metric that addresses the suitability of the QoS requirements of a flow for a certain $\mathrm{AP}$, without affecting the other flows active in the network.

- To exploit the SDN concept for implementing a finegrained control of the stations' flows, which is suitable for large networks.

- To largely enhance our previous work published in [4] in the facets described in the introduction section. 




Fig. 1 Controlling Wi-Fi network according to SDN Management Model.

\section{SDN-BASED WI-FI MANAGEMENT FRAMEWORK}

The algorithm presented in this paper exploits the flexibility of SDN to provide a dynamic AP selection process that takes into account the QoS requirements of wireless users, as well as the overall capacity of the network. To better explain the functionality of the proposed algorithm, we first need to present the developed SDN management framework upon which this algorithm has been designed.

We consider the scenario of a large Wi-Fi network of $N$ APs with heavy data traffic and heterogeneous wireless user demands, as introduced in Section I. However, according to SDN, all Wi-Fi APs providing different applications are centrally managed by a SDN controller, as illustrated in Fig. 1. The controller is capable of running multiple algorithms simultaneously and applies the resulting configurations to the managed Wi-Fi network.

The framework proposed in this paper is based on the architecture presented in [27] which has been developed in the context of the EU H2020 Wi-5 (What to do With the Wi-Fi Wild West) project [28]. The Wi-5 project addresses spectrum congestion in Wi-Fi networks by adopting SDN as an approach to manage Wi-Fi APs.

The Wi-5 architecture has been designed to provide efficient radio resource management solutions for the following use cases: (1) Airport/train station to address the typical network deployments found in public places, where a certain number of Wi-Fi APs are used to provide coverage to users in the area; (2) Dense apartment building that corresponds to a Wi-Fi scenario where the tenants in each apartment arrange their broadband connection independently; (3) Pico-cell street deployment to locally provide a high capacity in public areas like shopping streets and squares with terraces; (4) Large home or Small Office/Home Office (SOHO) that is a common Wi-Fi scenario, which has evolved beyond a simple deployment around a single and central AP. In this use case the users are responsible for managing their own local Wi-Fi access network, which is connected to the high capacity broadband connection supplied by their Internet Service Provider (ISP); and (5) Community Wi$F i$, which allows operators to offer Wi-Fi network access to their on-the-go subscribers by using existing residential and
Small Medium Businesses (SMB) Wi-Fi infrastructure, if the owners of the infrastructure agree with the provision of the service. The use cases are described in more detail in [29].

The choice of SDN to build a Wi-Fi network management platform is justified by the centralised nature of this concept which offers the operators and any entity that manages a Wi-Fi AP, including households, an interface through which a cooperative spectrum utilisation policy could be agreed and implemented. Furthermore, it has been already proven that SDN is an effective way to achieve fast handovers, frequency selection, and power control in Wi-Fi networks [26]. Moreover, SDN offers flexibility and cross-layer management, as the central controller is able to obtain monitoring information about the status of the network and execute relevant algorithms to react accordingly while respecting the requirements of the wireless users, as shown by the work in [4], [5] and [30]. It is worth mentioning that Wi-5 is currently developing a SDN framework that extends the capabilities of OpenFlow and relaxes its limitations to support the monitoring of Wi-Fi networks, the QoS requirements of wireless applications, and the configuration of Wi-Fi APs [31].

A series of tools that facilitate information gathering from the radio environment will be included in the SDN framework, taking also into consideration possible limitations of the measurement processes. For example, the delay and overhead incurred when a monitoring process is triggered will be measured in order to reduce their impact in real-time assessments. Further details on this implementation can be found in [31] and [32].

The radio resource management solutions included in the framework can run as applications on top of the controller and are triggered according to the network needs. Specifically, the applications that can be triggered by the SDN controller and considered in the framework proposed in this paper are Channel Assignment and AP Selection.

Although the main objective of this paper is to tackle efficient AP selection suitable for a large Wi-Fi network, it is also crucial for addressing the optimization of spectral efficiency and capacity in terms of the available bandwidth of the network in order to better guarantee the QoS requirements of the wireless users. Motivated by this consideration, we therefore, also include in our SDN-based framework a channel assignment algorithm to address the reduction of spectrum congestion and the magnitude of interference between the APs available in the Wi-Fi network.

The channel assignment algorithm is implemented on top of our SDN controller, and takes into account the following factors: (i) the Wi-Fi system properties (e.g. IEEE 802.11's standard channel characteristics); (ii) the logical network topology (i.e. APs' distribution throughout the network); and (iii) the desired resource management criteria (e.g. the assigned channel configuration, interference related QoS or handover requirements). The approach upon which this channel assignment is based has been presented and assessed in [5]. The performance analysis in [5] shows that this channel assignment algorithm provides lower interference, better SINR and higher spectral efficiency within the network, compared to the state of the art techniques. 


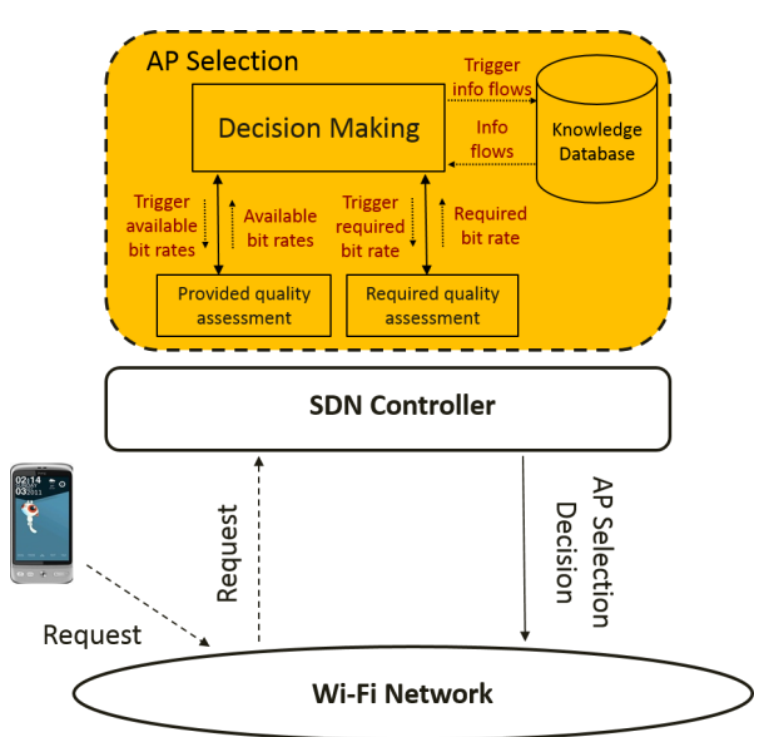

Fig. 2 AP Selection Approach Using SDN Concept.

The reader is referred to Appendix 1 for the analytical details and an analysis of the achievements of this solution in terms of spectral efficiency, which are exploited to strengthen the AP selection algorithm proposed here.

After the execution of the channel assignment algorithm, it guarantees the optimal spectrum efficiency in the network. Afterwards, when receiving each station connection request redirected from the Wi-Fi network, the SDN controller triggers the AP selection algorithm running on the controller as illustrated in Fig. 2. Moreover, since the proposed algorithm is focused on the QoS served to the wireless user, the AP selection can adapt to a change to the QoS requirements, e.g. another AP is selected for the wireless user if the current $\mathrm{AP}$ can no longer provide the QoS requirements for a certain application. Hence, the AP selection is triggered when either a new user joins the network, or an existing user switches to another application implying a new flow with different QoS requirements.

The algorithm consists of performing an efficient AP selection for the flows by executing two main steps. For each new flow trying to connect to the network, it firstly needs to properly match the bit rate requirements with the achievable bit rate in each AP through the FF. Then, the algorithm needs to compute the effect, which the new flow causes to the rest of the flows connected to the network, which are defined as active flows from now on. The result of these steps allows the SDNbased controller to select for the new flow the AP characterized by the Network Fittingness Factor, which will be explained in detail in Section IV.

We, therefore, define the following modules depicted in Fig. 2, upon which the algorithm relies to achieve this dynamic AP selection strategy: Provided Quality Assessment (PQA), Required Quality Assessment (RQA), Knowledge Database (KD) and Decision Making (DM). The description of the modules implemented in the SDN-based framework is provided in the following subsections, while their roles in the algorithm will be explained in Section IV.

\section{A. Provided Quality Assessment}

The PQA module gives information on the bit rate that each AP of the network can achieve for a new station request, measured at the physical layer connection. The assessment is obtained by the computation of the link capacity available for each new flow in terms of the bit rate, which in turns depends on the channel bandwidth assigned to each AP, the measured inter-AP interference within the network, and the position of the station requiring the connection. The details of this computation will be provided in Section IV. The link capacity of an AP corresponds to the most efficient MCS to achieve the highest available bit rate under the interference level constraints. Moreover, we consider the MCSs computed by using the Orthogonal Frequency Division Multiple Access (OFDMA) approach, which has been adopted in most 802.11 protocols (e.g., $802.11 \mathrm{~g} / \mathrm{a} / \mathrm{n}$ ).

\section{B. Required Quality Assessment}

The RQA module translates the QoS requirements of a connection-requesting station into a bit-rate metric. The QoS requirements of the station depend on the nature of the data flow that the station is sending and receiving. These QoS requirements can be easily either proactively programmed into the SDN controller [33], or reactively inferred through QoS detection techniques such as Machine Learning (ML) strategies. In particular, the application of ML strategies to detect traffic in real-time has attracted significant attention in past works [34], [35]. For example, the ML-based classification approach presented in [35] achieves 99\% classification accuracy for VoIP traffic across the APs of their network. The source code designed for detecting traffic and, consequently, QoS requirements in [35] is available in a public repository ${ }^{1}$. Therefore, this capability can be easily implemented to work in our framework but the details of such an implementation are outside the scope of this paper. Hence, we assume that the information used by this process to compute the QoS requirements is available.

\section{Knowledge Database}

The KD keeps track of all the active flows connected to the network. Specifically, it stores the QoS requirements corresponding to each active flow and the link capacity in terms of the bit rate available for each active flow in the network. Such information will be used by the following DM process during the execution of the AP selection algorithm.

\section{Decision Making}

The DM module is triggered every time a new flow needs to be associated to an AP. It first collects from the PQA and RQA modules the available information, which depends on the radio environment. Then, it uses this information to calculate our FF metric for each AP according to the service it can provide for the new flow. Moreover, this process analyses the information 
retained in the $\mathrm{KD}$ to compute for each $\mathrm{AP}$ the change in the bit rates provided to the active flows, which might be caused by the acceptance of the new flow. Based on this information, the DM module determines the most suitable AP for each new flow characterized by the Network Fittingness Factor. Finally, it updates the KD with the link capacity for each new flow in the assigned AP. The details on the computation of the FF and Network Fittingness Factor are provided in the next section.

\section{AP SELECTION ALGORITHM}

This section provides a comprehensive description of our AP selection approach in which we will firstly elaborate the FF and Network Fittingness Factor concepts and then explain their use in the algorithm.

\section{A. Fittingness Factor}

The FF is a performance metric used by the AP selection algorithm to determine the suitability of an AP to satisfy a wireless user's QoS requirements. Since these QoS requirements are based on the characteristics of the data flow of each wireless user, the suitability of an AP to serve them takes into account the data bit rate that the flow requires and the data bit rate that an AP can deliver.

From a general perspective, we formulate the FF by extending a sigmoid function $U_{i, j}$, which denotes the bit rate achievable by the user $i$ from the AP $j$ for the requested bit rate. Note that with the sigmoid-based utility function, the value of $U_{i, j}$ increases as the bit rate for serving flow $i$ by $\operatorname{AP} j$ increases with respect to the bit rate required for flow $i$. Our aim in this paper is to target a more efficient association to an AP through the FF concept by penalising this value if the bit rate for serving flow $i$ by AP $j$ is much larger than the bit rate required for flow $i$ in order to address the suitability of an AP for a flow in terms of its available bit rate.

The FF metric computation is based on the formulation defined in [36]-[38], while the utility function $U_{i, j}$ used to depict the QoS perceived by user $i$ on AP $j$ is based on the formulation proposed in [39].

Specifically, for each flow $i$ and each AP $j$, a FF metric is calculated as follows:

$$
f_{i, j}=\frac{1-e^{-\frac{U_{i, j}}{\rho \cdot\left(R_{i, j} / R_{r e q, i}\right)}}}{\lambda}
$$

Here $U_{i, j}$ denotes the mentioned utility function defined by the following formula:

$$
U_{i, j}=\frac{\left[\rho \cdot\left(R_{i, j} / R_{\text {req }, i}\right)\right]^{\xi}}{1+\left[\rho \cdot\left(R_{i, j} / R_{\text {req }, i}\right)\right]^{\xi}}
$$

The parameters $\xi$ and $\rho$ in (1) and (2) reflect the different degrees of elasticity between the required bit rate and the bit rate available in the AP. In particular, as we will discuss in more detail below, the selection of these parameters influences the slope of the FF behaviour, which reflects the definition of the

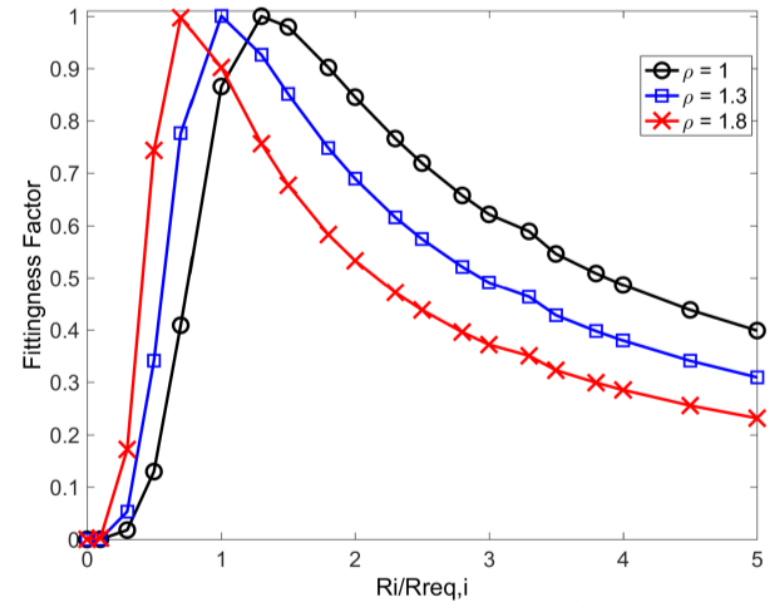

Fig. 3 Fittingness factor as a function of $R_{i} / R_{\text {req, } i}$ for different values of parameter $\rho$.

AP suitability for a certain flow with respect to the bit rate availability and the bit rate requirement. Moreover, $\lambda$ in (1) is a normalization factor used to ensure that the FF metric does not exceed 1 , and it is given by:

$$
\lambda=1-e^{-\frac{1}{(\xi-1)^{1 / \xi}+(\xi-1)^{(1-\xi) / \xi}}}
$$

$R_{\text {req, } i}$ in (1) and (2) denotes the bit rate required for flow $i ; R_{i, j}$ denotes the bit rate served to flow $i$ by AP $j$. Note that $R_{\text {req, } i}$ is obtained via the RQA module and $R_{i, j}$ is computed through the information obtained via the PQA. Specifically, let $\psi_{i, j}$ denote the SINR experienced by flow $i$ when associated with $\operatorname{AP} j . \psi_{i, j}$ is computed below at the location of the user requiring the connection of its flow $i$ to $\operatorname{AP} j[40]$ :

$$
\psi_{i, j}=\frac{g_{i, j} \cdot p_{j}}{\sum_{k \in N^{\prime}} g_{i, k} \cdot p_{k}+N_{0}}
$$

Here, $g_{i, j}$ is the channel gain from AP $j$ to flow $i, p_{j}$ is the transmit power of AP $j, N_{0}$ is the additive Gaussian white noise, and $N^{\prime} \subseteq N$ is the set of APs interfering with AP $j$ and therefore, affecting the SINR experienced by flow $i$. According to the $802.11 \mathrm{~g} / \mathrm{a} / \mathrm{n}$ standards, there exists a set of defined bit rate levels between $1 \mathrm{Mbps}$ and $54 \mathrm{Mbps}$ that can be provided by the APs. Each of these bit rate levels represents the link capacity $b_{i, j}$ between flow $i$ and AP $j$, which can be computed in the PQA module using $\psi_{i, j}$ through the Shannon-Hartley theorem. Therefore, $b_{i, j}$ can be expressed as:

$$
b_{i, j}=f\left(\psi_{i, j}, B W_{j}\right)
$$

Here, $B W_{j}$ is the bandwidth assigned to AP $j$ in $\mathrm{Hz}$. After the computation of $b_{i, j}$ provided by the PQA, the value of $R_{i, j}$ can be computed in the DM considering also the number $M$ of all the flows connected to AP $j$ available in the controller and the maximum capacity $C_{j}$ in bps available in AP $j$. Hence, $R_{i, j}$ can be expressed as the following function $g$ of all these parameters: 


$$
R_{i, j}=g\left(b_{i, j}, M, C_{j}\right)
$$

Further details on the computation of $R_{i, j}$ by making use of $b_{i, j}$ will be provided in subsection IV.C.

Fig. 3 plots the evolution of the FF values computed through (1) as a function of the ratio between the available bit rate served to a certain flow $i$ and its required bit rate defined in the figure as $R_{i}$ and $R_{\text {req, }}$, respectively. In this example we have selected $\xi=5$ because this value allows a smooth decrease of the FF when the available bit rates gradually become larger than the requirements. In fact, the greater $\xi$ 's value, the closer the FF to the sigmoid function [36]. In this figure we aim to illustrate how the selection of $\rho$ affects the behaviour of the FF, by considering three different cases with $\rho=1,1.3$ and 1.8, respectively.

The figure illustrates that the case $\rho=1$ exhibits the maximum value of the FF when the available bit rate is greater than the requirement (i.e., when $R_{i} / R_{\text {req, } i}$ is approximately 1.3 ). The case $\rho=1.3$ depicts the maximum value of the FF when the assignment equals the requirement (i.e., when $R_{i} / R_{\text {req }, i}=1$ ). Finally, the condition $\rho=1.8$ exhibits the maximum FF value when the available bit rate is lower than the requirement (i.e., when $R_{i} / R_{\text {req }, i}$ is approximately 0.7$)$. This means that the $\rho$ parameter defines the degree of suitability between the requirements and APs, provided by the FF through (1). The effect of the selection of the $\rho$ parameter in the performance results will be illustrated in Section VI.

\section{B. Network Fittingness Factor}

Although the FF described previously can assist in finding a suitable AP to serve each new flow, this metric does not reflect the effect of a potential association between an AP and a flow on the rest of the network. In reality, when a wireless user is associated with an AP, the overall network capacity may decrease in the sense that serving the new flow might affect the performance of part of the network.

We, therefore, define another parameter called Network Fittingness Factor (net $t_{f}$ ), which relies on the Standard Deviation Function $(\sigma)$. In detail, the Standard Deviation Function defines the variation in terms of the average FF that might result when an AP $j$ starts serving a new flow $i$.

For each $\mathrm{AP} j$, the available bit rate served to each active flow is recomputed through (6) by considering the effect caused by the connection of new flow $i$. Based on the new values of the bit rates, the FFs of the active flows are then updated through (1). Finally, the standard deviation is calculated as following:

$$
\sigma_{i, j}=\sqrt{\frac{\sum_{k=1}^{K}\left(f_{k, j}-\bar{f}_{J}\right)^{2}}{K}}
$$

where $\bar{f}_{J}$ is defined as following:

$$
\overline{f_{J}}=\frac{1}{K} \sum_{k=1}^{K} f_{k, j}
$$

In (7) and (8), $K$ represents the number of all active flows in AP $j$, which includes the previous flows active in the AP with their FFs updated, and the new flow $i$.

Given that there are $N$ APs available for selection to serve the new flow $i$, the Network Fittingness Factor is used to optimise the following parameters: (i) the FF metric of the AP serving the new data flow, and (ii) the standard deviation factor that maintains the performance of the overall network as much as possible, in order to determine the most suitable AP. This optimisation is formulated below:

$$
\begin{array}{r}
\text { net }_{f_{i}}=\arg \max _{j \in\{1, \ldots, N\}}\left\{F_{i, j}\right\} \\
\text { where } F_{i, j}=f_{i, j}\left(1-\sigma_{i, j}\right)
\end{array}
$$

Hence, net $_{f_{i}}$ computed through (9) aims to optimise the individual performance of the new flow to the associated AP by maximizing its FF, while trying to safeguard the overall network performance by minimizing the impact on the other active flows through the standard deviation. Note that for an AP with no other active flows, its standard deviation value is 0 .

\section{AP Selection Algorithm}

The objective of this algorithm is to find a suitable AP among the $N$ APs composing the Wi-Fi network with which the wireless user could be associated such that: (i) the AP provides the QoS performance requested by the new flow, and (ii) the AP association should safeguard the overall network performance.

The KD stores for each $\mathrm{AP} j$ the following sets: (i) $R_{\text {req }}^{j}$ that includes the QoS requirements in terms of bit rates corresponding to its active flows; and (ii) $B^{j}$ with the link capacities in terms of bit rates of the active flows computed through (5). As it is depicted in Fig. 2, each time a new flow triggers the request of an AP allocation, the DM module implemented in the SDN-based controller makes use of:

- the quality information obtained from the RQA that provides the bit rate required by the new flow;

- the link capacity in terms of the bit rate for the new flow from each AP in the network obtained from the PQA;

- the QoS requirements and the available bit rates for each active flow in the network from the KD.

Algorithms 1 and 2 depict in detail the running sequence of these interactions during the execution of the algorithms.

Firstly, to find the best AP to serve a new flow $i$, the DM module starts by collecting the required bit rate $R_{\text {req,i }}$ from the RQA (line 1 in Algorithm 1 below). Then, for each AP $j$, it collects from the PQA the link capacity $b_{i, j}$ in terms of the bit rate, which the AP can provide and is computed using (5) (line 4 in Algorithm 1). It then computes the set $R^{j}$ of $\mathrm{AP} j$, which includes the available bit rate for the new flow together with all the updated bit rates available to serve the existing active flows in $\mathrm{AP} j$, where the updated bit rates take into account the effect caused by the possible connection of flow $i$ (line 5 in Algorithm 1). Note that $R^{j}$ is computed through Algorithm 2, which will be explained below. 
The DM module then gets the set $R_{\text {req }}^{j}$ stored in the KD, and adds $R_{\text {req }, i}$ in $R_{\text {req }}^{j}$ (lines 6 and 7 in Algorithm 1). Afterwards, it computes all the FF values achieved for all the flows (including flow $i$ ) in $\mathrm{AP} j$ based on the bit rates in $R^{j}$ and $R_{\text {req }}^{j}$, respectively, using (1) and stores these values in set $F S$ (lines 8-12 in Algorithm 1). Hence, the DM can use (7) to compute the Standard Deviation Function $\left(\sigma_{i, j}\right)$ for AP $j$ based on these computed values in FS (line 13 in Algorithm 1). Afterwards, the DM module calculates value $F_{i, j}$ for AP $j$ using (9) and stores it in set $N F$ (lines 14 and 15 in Algorithm 1). Having completed the computation of each $F_{i, j}$ with $j \in\{1, \ldots, N\}$ (between lines 3 and 16 in Algorithm 1), it determines the most suitable AP for flow $i$ based on $n^{2} t_{f_{i}}$ in (9) (line 17 in Algorithm 1). Finally, the DM updates the sets $R_{\text {req }}$ and $B$ corresponding to the selected AP, which include the required bit rate and the link capacity for new flow $i$, respectively, and stores them in the KD (line 18 in Algorithm 1).
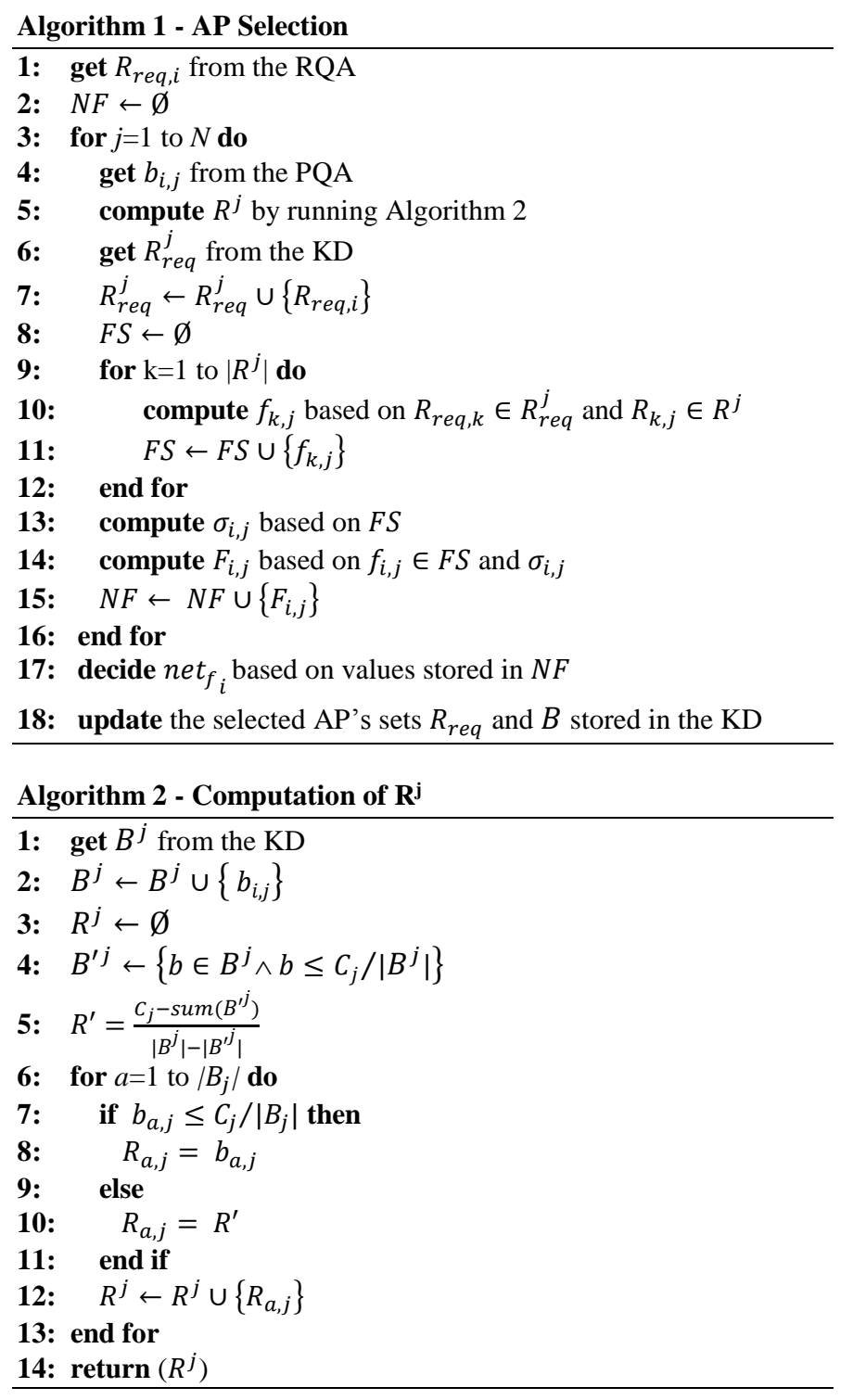

Focusing now on the computation of $R^{j}$, as defined in Algorithm 2, the DM module firstly gets the stored set $B^{j}$ from the $\mathrm{KD}$, which contains the link capacities (in terms of bit rates) of all the active flows in AP $j$, and adds $b_{i, j}$ in $B^{j}$ (lines 1 and 2 in Algorithm 2). Afterwards, the DM module computes $R^{j}$ by considering that all the flows associated with $\operatorname{AP} j$ can share the access time (lines 3-13 in Algorithm 2).

Let us focus on the computation of the available bit rate $R_{a, j}$ served to a flow $a$ by AP $j$, as stated in lines 4-11 of Algorithm 2 and specified by (6). The upper bound of $R_{a, j}$ is defined by dividing the total capacity $C_{j}$ (in bps) of AP $j$ by the number of its active flows (note that this number indicated by $M$ in (6), is derived in Algorithm 2 through the cardinality of set $B_{j}$ ). A set $B^{\prime j}$ including the flows with their link capacities not higher than the upper bound is created in order to compute the average capacity for all the other flows. Then, the available rate served to the flow in AP $j$ and called $R^{\prime}$ is equally shared with the other flows with their link capacities greater than the upper bound (see lines 4 and 5 of Algorithm 2). Finally, if the link capacity of a flow is lower than the upper bound, the available rate served to the flow corresponds to its link capacity (see lines 7 and 8 of Algorithm 2). Otherwise, the available rate served to the flow corresponds to $R^{\prime}$ (see lines 9 and 10 of Algorithm 2).

We now look into the complexity of our algorithms. Let $L$ be the number of all the active flows in the network at a certain time instant $t$, which are equally distributed among the $N$ APs throughout the network. The first for cycle in the AP selection algorithm is called $N$ times (line 5 in Algorithm 1). During each of the $N$ iterations, firstly Algorithm 1 computes $R^{j}$, which calculates on average $L / N$ bit rates served to each flow in AP $j$ (lines 6-13 of Algorithm 2). Then, Algorithm 1 computes on average $L / N$ FF values (lines 9-13 of Algorithm 1). Therefore, the time complexity of our AP selection algorithm is linearly related to the number of flows and we can define its approximation as:

$$
O(N \cdot(L / N+L / N))=O(L)
$$

\section{EVALUATION SCENARIO AND METRICS}

To evaluate our proposed AP selection algorithm, we use MATLAB to simulate the SDN-based controller in a dense WiFi environment consisting of 50 APs randomly deployed in an area of $1050 \times 1050 \mathrm{~m}^{2}$ at a minimum distance of 75 meters among them, with a transmit power of $25 \mathrm{dBm}$, and a free space path loss with exponent 2 . The values of $B W_{j}$ in (5) and $C_{j}$ in (6) are set, respectively, as $20 \mathrm{MHz}$ and $54 \mathrm{Mbps}$ for all the APs composing the network.

In the evaluation, we first simulate the SDN controller running the channel assignment algorithm to apply the optimal RF configuration to the Wi-Fi network. Once the channel assignment configuration is applied, we simulate a dense wireless environment, in which a new downstream flow trying to connect to the network is created every 3 minutes. The new flow, representing a new wireless user, or an existing user with new QoS requirements, is created in a random position within 
the designated area. The evaluation is stopped when the number of flows connected to the network reaches 1000. Finally, in the evaluation we simulate the SDN controller that executes the AP selection algorithm every time when a new flow tries to join the network. Note that this scenario and its selected settings are representative of a general dense environment, which addresses all the use cases considered in the context of the Wi5 project and summarised in Section III, and that can be easily adapted to such use cases.

To benchmark the performance of the proposed AP selection algorithm, two strategies presented in the literature are considered as candidates that address the same problem analysed in this paper. Specifically, we compare our AP selection algorithm against the following reference strategies found in the state of the art:

1) An AP selection algorithm that associates each new flow with the least loaded AP, which provides a sufficient RSSI based on the QoS requirements as proposed in [6]. We consider this AP load-based solution because it also targets a similar centralised approach relying on SDN. By comparing our algorithm to this scheme, we demonstrate that the FF metric allows us to achieve better performance against such an AP selection strategy that also tries to optimize the load balance of the APs.

2) An AP association solution where the selection criteria are based on the data rate an AP can achieve as proposed in [9]. We consider this data rate-based strategy because it is a common policy used to decide whether to associate with an $\mathrm{AP}$, assuming that each flow shares the access time equally with the others assigned to the same AP.

The evaluation of our approach against the above two strategies focuses on the following performance metrics:

- Average Blocking Probability: This is the average probability to deny the connection of a flow when it decreases the average satisfaction (defined below) that the selected AP guarantees to the connected flows, by a certain percentage. This probability is updated each time when a new flow is associated to an AP of the network.

- Average Data Bit Rate: It represents the average data rate in terms of bps that the assigned APs serve to the flows connected to the network. Specifically, for each new flow $i$ associated to an $\mathrm{AP} j$, we firstly compute the data bit rate $d_{i}$ served to the flow as follows:

$$
d_{i}=\left\{\begin{array}{lr}
R_{\text {req }, i} & \text { if } R_{i, j} \geq R_{\text {req }, i} \\
R_{i, j} & \text { otherwise }
\end{array}\right.
$$

Then, we consider as a performance metric the data rate averaged for all the flows active in the network on the simulation.

- Average Satisfaction: This is the average percentage of flows connected to the network with their served data bit rates (i.e., $d_{i}$ for flow $i$ ) higher than or equal to their given requirements (i.e. $R_{\text {req }, i}$ for flow $i$ ). This percentage is updated each time when a new flow is associated to an AP of the network.

- Percentage of Flows with Good Mean Opinion Score (MOS): This metric addresses the QoE of an application provided to a certain flow as the perceived acceptability from the user's point of view [41]. Specifically, in this paper we consider the MOS as a metric that provides the human user's view of the quality of the network. The MOS is an arithmetic mean of all the individual scores achieved by the result of subjective tests, and can range from 1 (worst) to 5 (best). The meaning of each score is illustrated in Table I in terms of quality and impairment. In the context of our analysis, we show the percentage of flows that achieve at least a Good quality at the end of the simulation.

The QoS requirements of the active flows of the stations trying to connect have been randomly generated from a set of bit rates that vary between $40 \mathrm{kbps}$ and $5 \mathrm{Mbps}$. We have chosen these values in order to represent the minimum bit rates required for common online applications (i.e., VoIP and video streaming on YouTube and Netflix), which are illustrated in Table II. Specifically, for each application in the table, we illustrate: (i) the bit rate requirements, (ii) the achievable MOS when assigning these requirements, (iii) the corresponding quality perceptible by the end-user, and (iv) the impairment corresponding to the quality. In the case of VoIP, we have considered $40 \mathrm{kbps}$ and $50 \mathrm{kbps}$, which are the approximate bit rate requirements that provide a Good MOS when the G.729 codec and G.723.1 codec are used, respectively ${ }^{2}$. Note that the Good MOSs for VoIP illustrated in the table also take into account other parameters such as the codec sample interval and the number of packets sent per second. On the other hand, the bit rate requirements that we address in our algorithm are high enough to allow to reach the Good MOS illustrated in the table.

In the case of video streaming, the minimum bit rate requirement for watching videos on YouTube is $500 \mathrm{kbps}$, and it is $1 \mathrm{Mbps}$ in the case of premium content such as movies, TV shows and live events ${ }^{3}$; and finally, $5 \mathrm{Mbps}$ is the minimum bit rate recommended for High Definition (HD) quality videos on Netflix ${ }^{4}$. Note that in the case of video streaming, the MOS is affected mostly by the stalling effect, which occurs when the video bit rate is larger than the available data rate [42]. This means that the video buffer is emptied causing the so-called freezing effect on the video until the buffer is filled again. Therefore, providing the suggested requirements for the video streaming applications allows the minimisation of the stalling effect. In [42] the authors demonstrate that the reduced number of stalling effects achievable through high data rates allows them to obtain the Good MOSs values illustrated in Table II in the case of video streaming.

\footnotetext{
${ }^{4}$ https://help.netflix.com/en/node/306 (accessed November 2016).
}

\footnotetext{
http://www.cisco.com/c/en/us/support/docs/voice/voice-quality/7934bwidth-consume.html (accessed November 2016).

3 https://support.google.com/youtube/answer/78358?hl=en-GB (accessed November 2016).
} 
TABLE I

MEAN OPINION SCORE - MOS

\begin{tabular}{ccc}
\hline \hline MOS & Quality & Impairment \\
\hline 5 & Excellent & Imperceptible \\
4 & Good & Perceptible but not annoying \\
3 & Fair & Slightly annoying \\
2 & Poor & Annoying \\
1 & Bad & Very annoying \\
\hline \multicolumn{3}{c}{ TABLE II } \\
& BIT RATE REQUIREMENTS AND MOS
\end{tabular}

\begin{tabular}{ccccc}
\hline \hline Application & Bit rate & MOS & Quality & Impairment \\
\hline VoiP G.729 & $40 \mathrm{kbps}$ & 3.92 & & \\
VoiP G.723.1 & $50 \mathrm{kbps}$ & 3.9 & & Perceptible \\
YouTube & $500 \mathrm{kbps}$ & 4.5 & Good & but not \\
Premium YouTube & $1 \mathrm{Mbps}$ & 4.5 & & annoying \\
Netflix HQ & $5 \mathrm{Mbps}$ & 4.5 & & \\
\hline
\end{tabular}

The minimum bit rate included in Table II represents the $R_{\text {req, } i}$ of an active flow $i$ presented in (1). Moreover, for the sake of simplicity, we do not consider possible effects of the interference from wireless devices using VoIP in the uplink direction. This is a reasonable assumption, since this paper focuses on demonstrating how the selection of the most suitable AP addresses both QoS performance and spectrum efficiency of the flows.

In addition, we have focused on two cases illustrated in Fig. 3 for two different experiments:

1) In the first experiment we have considered $\xi=5$ and $\rho=$ 1.3 in (1), which corresponds to maximizing the FF when the available bit rate equals the minimum bit rate requirements of the application (see Fig. 3). Therefore, in the first experiment, net $_{f_{i}}$ computed through (9) aims to optimise the individual performance of the new flow on the selected AP by maximizing its FF, which exhibits the maximum value when the assigned bit rate equals the required bit rate.

2) In the second experiment we have considered $\xi=5$ and $\rho$ $=1$ in (1), which corresponds to maximizing the FF value for more efficient APs in terms of the bit rates (see Fig. 3). Hence, in the second experiment, net $_{f_{i}}$ aims to optimise the individual performance of the new flow on the associated AP by maximizing its FF, which exhibits the maximum value when the available bit rate is greater than the required bit rate.

Therefore, through these experiments, we aim to analyse also the trade-off between the selection of the parameters in (1) and the achieved performance results.

\section{EVALUATION RESUlTS}

Based on the configuration previously described, our FFbased algorithm and the other existing strategies for maximizing the achievable data rate and AP load were executed in the controller every time when a new user tried to join the network, or an active user needed a new flow with different QoS requirements.
The results achieved for the first experiment are illustrated from Fig. 4 to Fig. 7. In detail, Fig. 4 shows the performance results in terms of the number of flows, achieved by our proposed algorithm and by the data rate-based and AP loadbased strategies. The simulation considers blocking a new flow when it decreases the average satisfaction, which the selected AP guarantees to the active flows, by $10 \%$.

The performance in terms of the blocking probability is illustrated in Fig. 4(a) where we can observe that our AP selection algorithm reduces the blocking probability compared with the data rate-based and AP load-based solutions. In particular, the figure illustrates that when the number of flows reaches 1000, AP selections based on the data rate and AP load result in around $10 \%$ of the flows being blocked. For the same number of flows, the AP selection based on the FF results in approximately $7 \%$.

Fig. 4(b) shows the performance in terms of the average satisfaction as a function of the number of the flows connecting to the network. This figure illustrates that the FF-based algorithm offers significantly better flow satisfaction than the data rate-based and load-based solutions. Specifically, when the number of flows reaches 1000 our AP selection algorithm outperforms the data rate-based strategy by around $18 \%$, and the AP load-based solution by around $14 \%$.

Fig. 4(c) illustrates the average data rate available for each flow as a function of the number of the flows that join the network. In this case, the FF-based algorithm again outperforms the data rate-based and load-based solutions.

The above better performance results achieved by our algorithm can be attributed to the optimisation approach to finding a suitable AP while not degrading the overall performance of the network.

Fig. 5 illustrates the performance achieved as a function of the number of flows considering the blocking of a new flow when it decreases the average satisfaction, which the selected AP guarantees to the active flows, by $20 \%$. In this case, the condition that determines the blocking allows accepting more flows into the network compared to the previous case.

Fig. 5(a) shows that although this condition enables a significant improvement in terms of the blocking probability for all the strategies, our AP selection algorithm still outperforms the data rate-based and load-based solutions.

Fig. 5(b) shows that the performance in terms of the average satisfaction as a function of the number of flows connecting to the network slightly decreases for all the strategies compared to the previous case. Nevertheless, our solution still delivers the best results.

Fig. 5(c) also illustrates a slightly decrease of the performance results in terms of the average data rates available for the flows. This result was expected, as the condition that drives the blocking probability allows the algorithm to accept a greater number of flows and hence decreases the overall performance of the network. However, our algorithm continues to outperform the data rate-based and load-based solutions.

Fig. 6 shows the performance results in terms of the percentage of flows that reach at least a Good MOS, achieved 


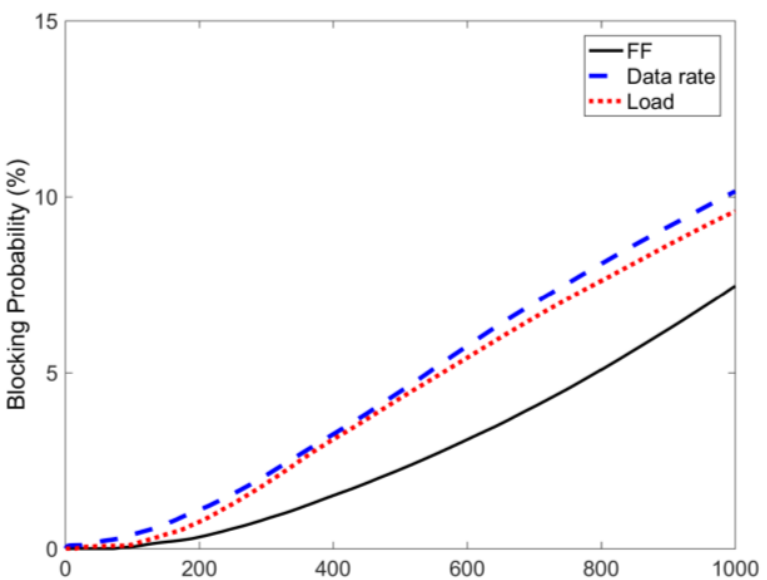

(a)

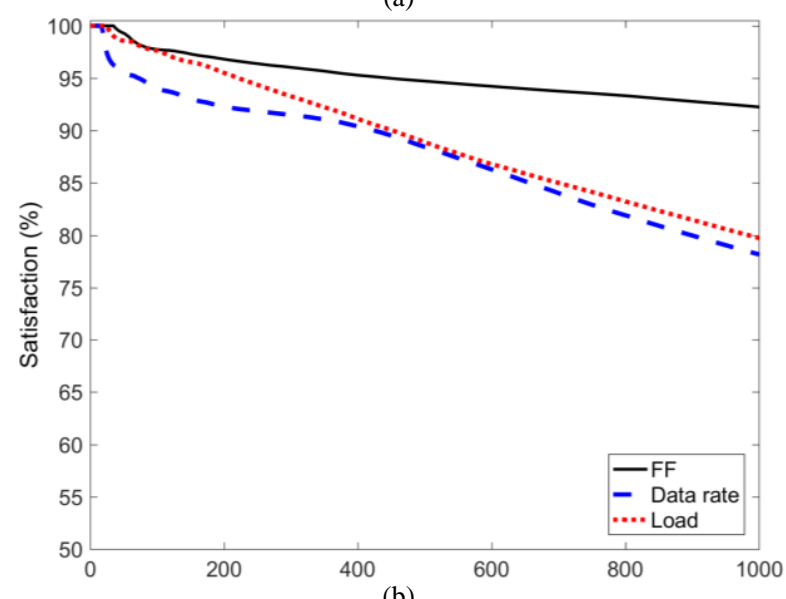

(b)

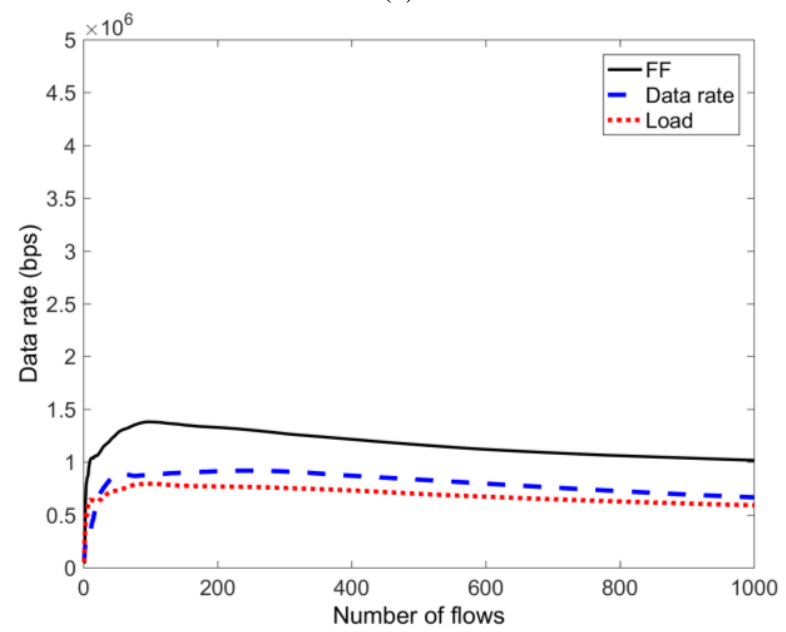

(c)

Fig. 4 Performance results when the average satisfaction is decreased by $10 \%$. (a) Blocking probability, (b) Satisfaction, (c) Data rate.

by our proposed algorithm and by the data rate-based and AP load-based strategies.

The left hand side of the figure illustrates the performance achieved by blocking a new flow when it decreases the average satisfaction by $10 \%$. The performance obtained by blocking a new flow when it decreases the average satisfaction by $20 \%$ is shown on the right hand side. The figure illustrates that in both analyses our AP selection algorithm outperforms the data ratebased and load-based solutions.

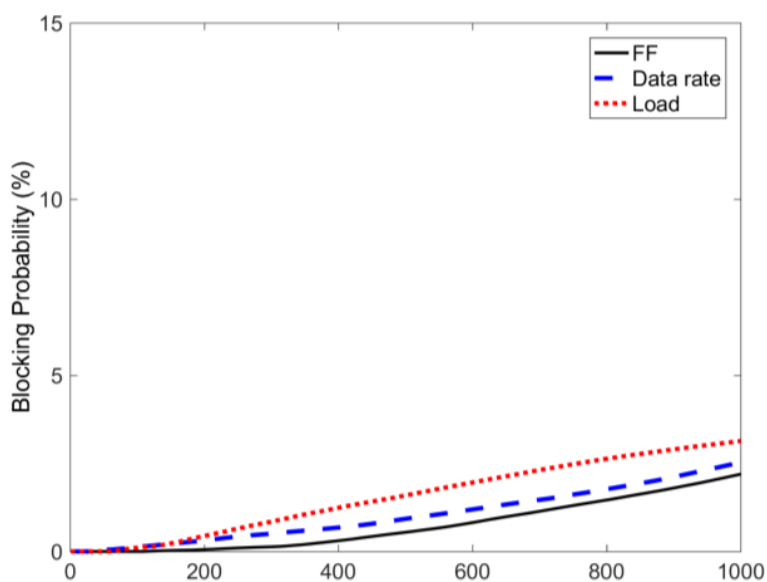

(a)

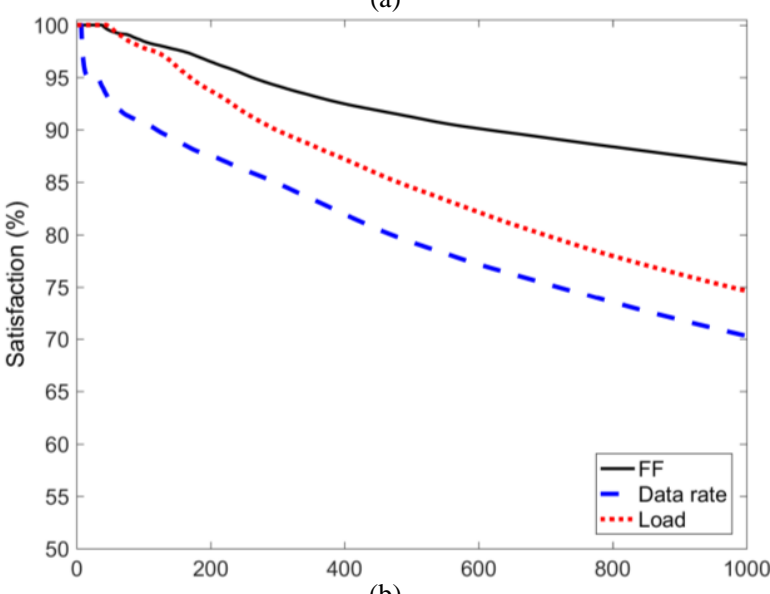

(b)

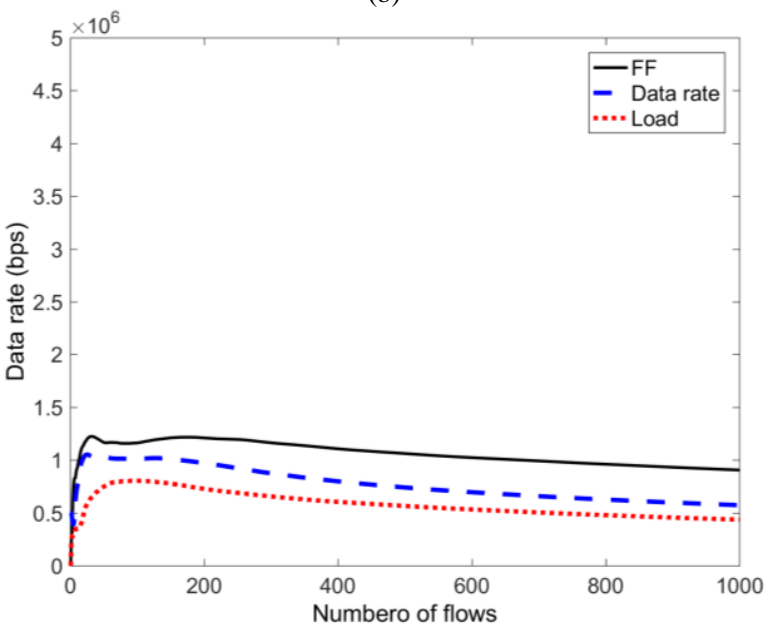

(c)

Fig. 5 Performance results when the average satisfaction is decreased by $20 \%$. (a) Blocking probability, (b) Satisfaction, (c) Data rate.

Finally, Fig. 7 summarizes the gains achieved by the FFbased algorithm over both the data rate-based and AP loadbased algorithms in terms of the average satisfaction as a function of the number of flows. This analysis considers blocking a new flow when it decreases the average satisfaction by $20 \%$, which is deemed as a better condition to balance the trade-off between the blocking probability and average satisfaction. In fact, as we have shown, this condition allows to decrease the blocking probability for all the strategies at the 


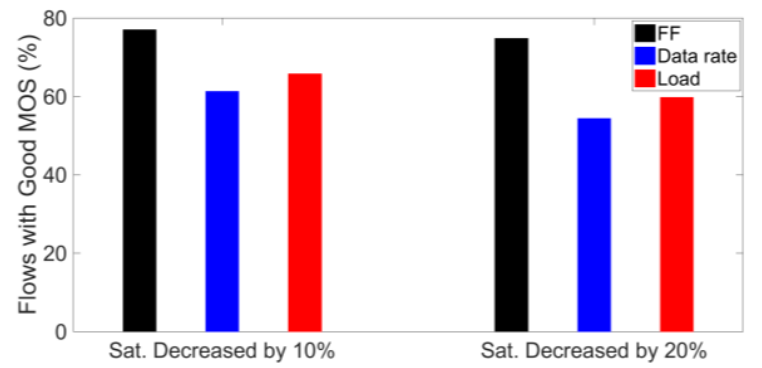

Fig. 6 Performance results in terms of the percentage of flows with at least Good MOS when $\rho=1.3$.

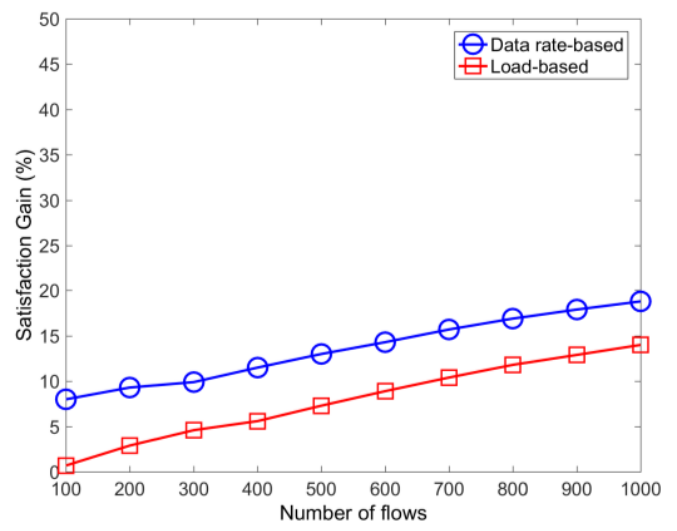

Fig. 7 Gains in terms of satisfaction achieved through the FF-based algorithm as a function of the number of flows when $\rho=1.3$.

expense of slightly degrading performance in terms of the satisfaction and data rate. For instance, in the case of our FFbased algorithm for 1000 flows we can observe an improvement of around $70 \%$ in terms of the blocking probability at the expense of reductions of around $6 \%$ and $11 \%$ for the satisfaction and the data rate, respectively, in comparison with the results illustrated in Fig. 4.

Fig. 7 shows that the FF-based algorithm achieves an improvement on the data rate-based approach in terms of the satisfaction, ranging from $8 \%$ for 100 flows to $18 \%$ in the case of 1000 flows. The improvement accomplished by the FF-based algorithm over the AP load-based approach ranges from $1 \%$ for 100 flows to $14 \%$ in the case of 1000 flows.

For the second experiment, we considered the setting $\rho=1$ in (1), which corresponds to the condition of maximizing the FF value for more efficient APs in terms of the bit rate. Specifically, Fig. 3 shows that the case $\rho=1$ means that the FF reaches its maximum value when the available bit rate is approximately 1.3 times the required bit rate.

For this experiment we measured the gains obtained from our FF-based algorithm over both the data rate-based and AP loadbased solutions in terms of the average satisfaction. The results are presented in Fig. 8. These results show that the FF-based algorithm outperforms the data rate-based solution within a range between $9 \%$ and $21 \%$. The figure also shows that the FFbased algorithm outperforms the load-based algorithm in terms of the satisfaction by a margin that varies between $2 \%$ and $16 \%$.

Note that in this case, the gains achieved by our algorithm are improved in comparison with those in Fig. 7. On the other

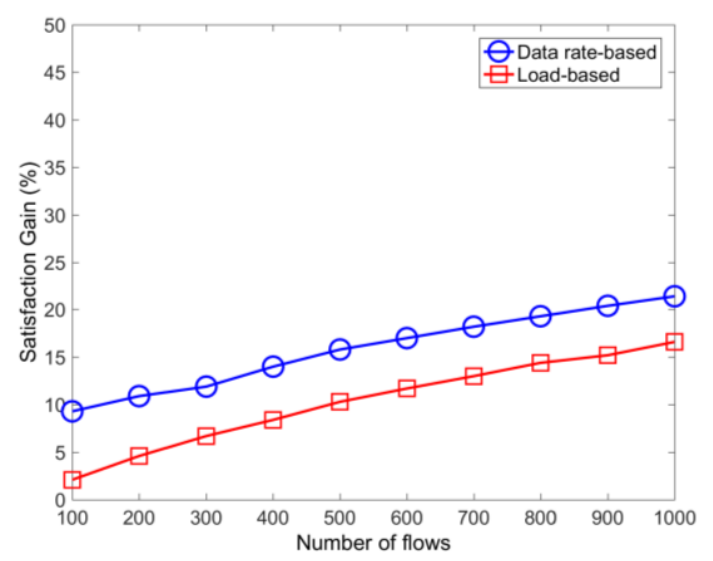

Fig. 8 Gains in terms of satisfaction achieved through the FF-based algorithm as a function of the number of flows when $\rho=1$.

hand, this condition in (1) affects the performance result of our algorithm in terms of the blocking probability. Specifically, for instance, when $\rho=1.3$ in (1) and the number of flows reaches 1000 , the AP selection based on FF achieves a blocking probability of approximately $2 \%$ (see Fig. 5(a)); whereas this value is around $3 \%$ when $\rho=1$. Note that $\rho=1$ means that the maximum value of the FF is reached when the available served bit rate is greater than the requirement, so trying to provide the maximum FF to a flow increases the blocking probability.

This demonstrates that by adjusting the Network Fittingness Factor through (9), a trade-off can be made for the optimisation between the blocking probability and the users' satisfaction. However, the setting $\rho=1.3$ in (1), which is the condition maximizing the $\mathrm{FF}$ when the bit rate assignment equals to the requirement, outperforms the state of the art, in terms of both the blocking probability and users' satisfaction.

\section{CONCLUSIONS AND FUTURE WORKS}

In this paper we have proposed a centralised network management framework for AP selection based on SDN. The framework presented in this paper implements an innovative algorithm that uses novel AP selection metrics that inherently consider the heterogeneity of the requirements for the different stations accessing the network. Moreover, the proposed algorithm takes into account the network capacity and the quality of the services provided to all the wireless users connected to the network.

The proposed framework has been evaluated via simulations to enable its comparison against two strategies found in the literature. These existing solutions maximize the achievable data rate and the load balance of the APs in the network, respectively. The evaluation results have shown that our algorithm achieves significant improvements over both solutions in terms of the blocking probability, assigned data rate, user satisfaction and MOS, when selecting $\rho=1.3$ in the FF formulation.

Our future work will consider improving the AP selection algorithm to include further QoS and QoE metrics and new applications such as online games. Moreover, motivated by the satisfactory results achieved through our SDN-based simulator 
presented in this paper, we will also consider the implementation and assessment of our AP selection algorithm in the SDN-based testbed being designed in the context of the Wi-5 project [31].

\section{APPENDIX 1 - CHANNEL ASSIGNMENT ASSESSMENT}

In our framework, we consider $N \mathrm{Wi}$-Fi APs that operate on $F$ RF channels. We define $G^{N \times N}$ as the network topology matrix where:

$$
g_{i j}=\left\{\begin{array}{cc}
1, & \text { average power strenght of } A P_{i} \text { around } A P_{j} \\
& \text { exceeds a threshold } \\
0, & \text { otherwise }
\end{array}\right.
$$

Then, we define $A^{F \times N}$ as the channel assignment matrix where:

$$
a_{i j}=\left\{\begin{array}{cc}
1, & \text { if channel } i \text { is assigned to } A P_{j} \\
0, & \text { otherwise }
\end{array}\right.
$$

We also define $I \in \mathbb{R}^{N \times F}$, which is the matrix of the interference predicted for $N$ APs and $F$ available channels, where $I_{i, j}$ is the interference level detected within the network as a result of assigning channel $j$ to AP $i$. Finally, we define $U$ as an objective function that represents the interference levels detected by all APs due to their current channel assignment configuration, and that can be formulated as following:

$$
W=G \times A^{T}, U=W . I
$$

Where ' $x$ ' represents the matrix multiplication and '.' denotes element-wise multiplication of the matrices. $U$ actually is obtained by taking into account the arrangement of the APs, reflected in $G$, and the channel assignment represented by $A$ alongside the actual impact of the channel selection at each AP from the interference point of view represented by $I$. Since $I$ is a matrix with real values $\left(I \in \mathbb{R}^{N \times \mathrm{F}}\right), U$ in (13) will also be a real matrix $\left(U \in \mathbb{R}^{N \times \mathrm{F}}\right.$ ). We can describe $U$ as an objective function which represents the magnitude of the interference in the whole system and encompasses the AP inter-relations, through $G$, and the scale by which APs are conflicting with each other in each channel represented by $G \times A^{T}$ as follows:

$$
U \equiv \overbrace{\begin{array}{r}
\text { represents the APs'interrelations } \\
\text { (with or without interference conflict) }
\end{array}}^{\begin{array}{c}
\text { encapsulates the scale by which APS } \\
\text { conflict in each specific channel }
\end{array}} A^{T}
$$

Finally, we define our optimised channel assignment matrix $A^{*}$ that provides the minimum interference levels throughout the network and can be obtained by minimizing the value of $U$.

Considering the scenario introduced in Section $\mathrm{V}$, we executed in the controller the above channel assignment algorithm with its further details presented in [5], to configure the RF channels of the APs forming the simulated network. We compared the achieved performance of our algorithm against the Least Congested Channel (LCC) selection mechanism, which is a common strategy found in many papers in the literature [42]. With the LCC approach, each AP acquires a suitable channel based only on the neighbouring APs' channels. Fig. 9 illustrates the performance in terms of the algorithms' interference level and spectral efficiency in $\mathrm{b} / \mathrm{s} / \mathrm{Hz}$.

Fig. 9(a) illustrates the interference levels achieved throughout the network using both our channel assignment approach and the LCC strategy. In the figure the upper and lower edges of the plotted boxes are the $25^{\text {th }}$ and $75^{\text {th }}$ percentiles of the values, and the median values are indicated by the central red lines. The values which we considered as outliers are indicated by blue dots in each case. The figure shows that our approach results in better interference levels compared to the LCC strategy. This includes a consistent reduction of the interference for all of the monitored values, including the outliers, which results in a $2 \mathrm{~dB}$ reduction in the average interference level in the network compared to the LCC approach.

Fig. 9(b) shows the corresponding achieved spectral efficiency for the channel assignment algorithm considered in our SDN-based framework against the LCC mechanism. The upper and lower sides of the depicted lines represent the range of the values from the $1^{\text {st }}$ to the $3^{\text {rd }}$ quartiles alongside the median value indicated at the middle of the lines. The outlier values are represented by the thin part of the lines.

The obtained result presented in this figure shows that our SDN-based algorithm outperforms LCC with a gain of 0.56 $\mathrm{b} / \mathrm{s} / \mathrm{Hz}$. In terms of the channel capacity that can be exploited to enhance our AP selection algorithm, this improvement is equal to extra 11.2 Mbps per used RF channel in the achievable physical layer data rate.

\section{REFERENCES}

[1] O. Galinina, et al., "5G Multi-RAT LTE-WiFi Ultra-Dense Small Cells: Performance Dynamics, Architecture, and Trends", IEEE Journal on Selected Area in Communications, vol. 33, no. 6, Jun. 2015.

[2] G. Judd and P. Steenkiste, "Fixing 802.11 access point selection, "ACM SIGCOMM Computer Comm. Review, vol. 32, no. 3, pp. 31, Jul. 2002.

[3] "Software-defined networking: The new norm for networks," Palo Alto, CA, USA, White Paper, Apr. 2012. [Online]. Available: https://www.opennetworking.org/images/stories/downloads/whitepapers/wp-sdnnewnorm.pdf.

[4] A. Raschellà, F. Bouhafs, M. Seyedebrahimi, M. Mackay, Q. Shi, "A Centralized Framework for Smart Access Point Selection based on the Fittingness Factor", International Conference on Telecommunications (ICT), Thessaloniki, Greece, 16-18 May, 2016.

[5] M. Seyedebrahimi, F. Bouhafs, A. Raschellà, M. Mackay, Q. Shi, "SDNBased Channel Assignment Algorithm for Interference Management in Dense Wi-Fi Networks", European Conference on Networks and Communications (EuCNC), Athens, Greece, 27-30 June, 2016.

[6] K. Sood, S. Liu, S. Yu, Y. Xiang, "Dynamic Access Point Association Using Software Defined Networking", International Telecommunication Networks and Applications Conference (ITNAC), Sydney, Australia,1820 Nov. 2015.

[7] H. Lee, et al., "Available bandwidth based association in IEEE 802.11 Wireless LANs," International Symposium on Modeling, analysis and simulation of wireless and mobile systems (MSWiM), Vancouver, Canada, 27-31 Oct. 2008.

[8] Y. Li-Hsing, J.-J. Li, and C.-M. Lin, "Stability and Fairness of AP Selection Games in IEEE 802.11 Access Networks", IEEE Transactions on Vehicular Technology, vol. 60, no. 3, pp. 1150-1160, Mar. 2011.

[9] X. Chen, et al., "Access Point Selection under QoS Requirements in Variable Channel-Width WLANs", IEEE Wireless Comm. Letters, vol. 2, no. 1, pp. 114-117, Feb. 2013. 


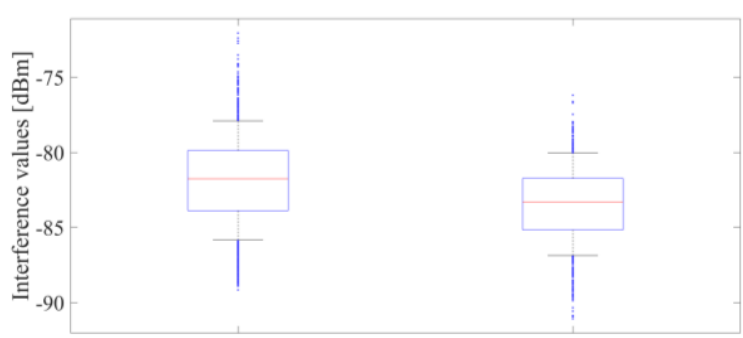

(a)

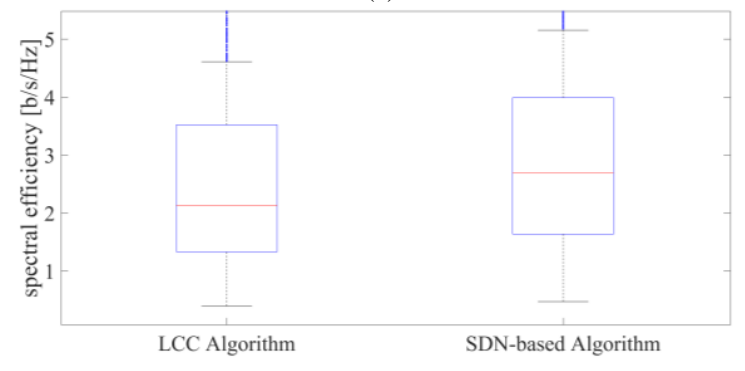

(b)

Fig. 9 Channel Assignment Algorithm Assessment. (a) Average interference levels, (b) Spectral efficiency.

[10] L. Chen, "A Distributed Access Point Selection Algorithm Based on Noregret Learning for Wireless Access Networks", Vehicular Technology Conference (VTC-Spring), Taipei, Taiwan, 16-19 May 2010.

[11] M. Liyanage, J. Chirkova, and A. Gurtov, "Access Point Selection Game for Mobile Wireless Users", International Symposium on a World of Wireless, Mobile and Multimedia Networks (WoWMoM), Sydney, Australia, 16-19 Jun. 2014.

[12] B. Bojovic, N. Baldo, and P. Dini, "A Neural Network Based Cognitive Engine for IEEE 802.11 WLAN Access Point Selection", Consumer Communications \& Networking Conference (CCNC), Las Vegas, Nevada, USA, 14-17 Jan. 2012.

[13] J. B. Ernst, S. Kremer, and J. J. P. C. Rodrigues, "A Utility Based Access Point Selection Method for IEEE 802.11 Wireless Networks with Enhanced Quality of Experience", IEEE International Conference on Communications (ICC), Sydney, Australia, 10-14 Jun. 2014.

[14] I. Malanchini, M. Cesana, and Nicola Gatti, "Network Selection and Resource Allocation Games for Wireless Access Networks", IEEE Transaction on Mobile Computing, vol. 12, no. 12, pp. 2427-2440, Dec. 2013.

[15] K. Sundaresan, K. Papagiannaki, "The Need for Cross-Layer Information in Access Point Selection Algorithms", ACM SIGCOMM conference on Internet Measurement (IMC), Rio de Janeiro, Brazil, 25-27 Oct. 2006.

[16] Y.-S. Chen, W.-H. Hsiao, and K.-L. Chiu, "A cross-layer partner-based fast handoff mechanism for IEEE 802.11 wireless networks", International Journal of Communication Systems, vol. 22, issue 12, pp. 1515-1541, Dec. 2009.

[17] A. Antonopoulos, et al., "Cross layer access point selection mechanisms for a distributed queuing MAC protocol", Telecommunication Systems vol. 53, issue 3, pp. 329-342, Jul. 2013.

[18] M. Abusubaih and A. Wolisz, "An optimal station association policy for multi-rate ieee 802.11 wireless lans," ACM Symposium on Modeling, analysis, and simulation of wireless and mobile systems, Chania, Crete Island, 22-26 Oct. 2007.

[19] J. Pang, et al., "Wifireports: improving wireless network selection with collaboration," Conference on Mobile systems, applications, and services, Krakow, Poland, 2009.

[20] A. Fujiwara, Y. Sagara, and M. Nakamura. "Access point selection algorithms for maximizing throughputs in wireless LAN environment", International Conference on Parallel and Distributed Systems (ICPADS), Hsinchu, Taiwan, 5-7 Dec., 2007.

[21] L.-H. Yen, T.-T Yeh, and K.-H. Chi, "Load Balancing in IEEE 802.11 Networks", IEEE Internet Computing, Vol. 13, Issue 1, Feb. 2009.

[22] N. McKeown, et al., "OpenFlow: enabling innovation in campus networks", ACM SIGCOMM Computer Communication Review, vol. 38, no, 2, pp. 69-74, 2008.
[23] K.-K. Yap, et al., "OpenRoads: Empowering Research in Mobile Networks", ACM SIGCOMM Computer Communication, Vol. 40 Issue 1, Jan. 2010.

[24] J. Schulz-Zander, et al., "OpenSDWN: programmatic control over home and enterprise WiFi", ACM SIGCOMM Symposium on Software Defined Networking Research, Santa Clara, CA, 14-17 Mar. 2016.

[25] R. Riggio, T. Rasheed, and F. Granell, "EmPOWER: A Testbed for Network Function Virtualization Research and Experimentation", IEEE SDN for Future Networks and Services (SDN4FNS), Trento, Italy 11-13 Nov. 2013.

[26] J. Schulz-Zander, L. Suresh, N. Sarrar, and A, Feldmann, "Programmatic Orchestration of WiFi Networks," USENIX Symposium on Networked Systems Design and Implementation (NSDI), Philadelphia, PA, USA, 1920 Jun. 2014.

[27] F. Bouhafs (Editor), "Wi-5 initial architecture", Deliverable D2.4 of Wi5 project, Dec. 2015, available at http://www.wi5.eu/.

[28] H2020 Wi-5 Project (What to do With the Wi-Fi Wild West), http://www.wi5.eu/

[29] I. Berberana (Editor), "Wi-5 use cases and requirements", Deliverable D2.3 of Wi-5 project, Dec. 2015, available at http://www.wi5.eu/.

[30] M. Seyedebrahimi, et al., "A Centralised Wi-Fi Management Framework for D2D Communications in Dense Wi-Fi Networks", Conference on Standards for Communications and Networking (CSCN), Berlin, German, 31 Oct.-2 Nov., 2016.

[31] J. Saldana (Editor), "Specification of Smart AP solutions version 1", Deliverable D3.2 of Wi-5 project, Dec. 2015, available at http://www.wi5.eu/.

[32] L. Sequeira, J. L. de la Cruz, J. Saldana, J. Ruiz-Mas, J. Almodóvar, "Building a SDN Enterprise WLAN Based On Virtual APs", IEEE Comm. Letters, Nov. 2016.

[33] R.Wallner, and R.Cannistra, "An SDN Approach: Quality of Service using Big Switch's Floodlight Open-source Controller", Proceedings of the Asia-Pacific Advanced Network 2013 vol. 35, p. 14-19, 2013.

[34] F. Rodríguez-Teja, C. Martinez-Cagnazzo, and E. G. Castro, "Bayesian classification: Methodology for network traffic classification combination," International Wireless Communications and Mobile Computing Conference (IWCMC), Caen, France, 28 Jun.-2 Jul., 2010, pp.

[35] T. T. T. Nguyen, G. Armitage, P. Branch, and S, Zander, "Timely and continuous machine-learning-based classification for interactive IP traffic," IEEE/ACM Transactions on Networking, vol. 20, no. 6, pp. 1880-1894, Dec. 2012.

[36] F. Bouali, O. Sallent, J. Pérez-Romero, R. Agusti, "Exploiting Knowledge Management for Supporting Spectrum Selection in Cognitive Radio Networks", 7th International Conference on Cognitive Radio Oriented Wireless Networks (CrownCom) 2012, Stockholm, Sweden, June. 2012.

[37] A. Raschellà, J. Pérez-Romero, O. Sallent, and A. Umbert, "On the use of POMDP for Spectrum Selection in Cognitive Radio Networks", International Conference on Cognitive Radio Oriented Wireless Networks (CROWNCOM), Washington DC, USA, 8-10 Jul. 2013.

[38] A. Raschellà, and A. Umbert, "Implementation of Cognitive Radio Networks to evaluate spectrum management strategies in real-time", Elsevier Computer Communications Vol. 79, Apr. 2016, Pages 37-52.

[39] L. Badia, M. Lindstrom, J. Zander, and M. Zorzi, "Demand and pricing effects on the radio resource allocation of multimedia communication systems," Global Comm. Conf. (GLOBECOM 2003), San Francisco CA, USA, 1-5 Dec. 2003.

[40] W. Li, et al., "Achieving Proportional Fairness via AP Power Control in Multi-Rate WLANs", IEEE Transaction On Wireless Communications, Vol. 10, No. 11, Nov. 2011.

[41] K. Piamrat, A. Ksentini, C. Viho, and J.-M. Bonnin, "QoE-aware Admission Control for Multimedia Applications in IEEE 802.11 Wireless Networks", Vehicular Technology Conference (VTC-Fall), Calgary, AB, Canada, 21-24 Sep., 2008.

[42] T. Hossfeld, R. Schatz, E. Biersack, and L. Plissonneau, "Internet Video Delivery in YouTube: From Traffic Measurements to Quality of Experience", Data Traffic Monitoring and Analysis: From Measurement, Classification, and Anomaly Detection to Quality of Experience, Springer Berlin Heidelberg, pp.264-301, 2013.

[43] M. Achanta, "Method and Apparatus for Least Congested Channel Scan for Wireless Access Points," US Patent No. 20060072602, Apr. 2006. 